\title{
Title: High-performance light-emitting diodes based on carbene-metal-amides
}

One Sentence Summary Spin-state inversion in rotationally-flexible linear carbene complexes enables high-performance solution-processed OLEDs.

Authors: Dawei $\mathrm{Di}^{1^{*}}$, Alexander S. Romanov ${ }^{2 *}$, Le Yang ${ }^{1 *}$, Johannes M. Richter ${ }^{1}$, Jasmine P. H. Rivett ${ }^{1}$, Saul Jones ${ }^{1}$, Tudor H. Thomas ${ }^{1}$, Mojtaba Abdi Jalebi ${ }^{1}$, Richard H. Friend ${ }^{1}$, Mikko Linnolahti $^{3 \dagger}$, Manfred Bochmann ${ }^{2 \dagger} \&$ Dan Credgington ${ }^{1 \dagger}$

\section{Affiliations:}

${ }^{1}$ Cavendish Laboratory, University of Cambridge, JJ Thomson Avenue, Cambridge, CB3 0HE, United Kingdom

${ }^{2}$ School of Chemistry, University of East Anglia, Earlham Road, Norwich, NR4 7TJ, United Kingdom

${ }^{3}$ Department of Chemistry, University of Eastern Finland, Joensuu Campus, FI-80101 Joensuu, Finland

†Correspondence to: djnc3@cam.ac.uk, m.bochmann@uea.ac.uk, or mikko.linnolahti@uef.fi.

\footnotetext{
* These authors contributed equally to this work.
} 


\begin{abstract}
:
Organic LEDs promise highly efficient lighting and display technologies. We introduce a new class of linear donor-bridge-acceptor light-emitting molecules, which enable solution-processed LEDs with near-100\% internal quantum efficiency at high brightness. Key to this performance is their rapid and efficient utilization of triplet states. Using time-resolved spectroscopy, we establish that luminescence via triplets occurs within $350 \mathrm{~ns}$ at ambient temperature, after reverse intersystem crossing to singlets. We find that molecular geometries exist at which the singlettriplet energy gap (exchange energy) is close to zero, such that rapid interconversion is possible. Calculations indicate that exchange energy is tuned by relative rotation of donor and acceptor moieties about the bridge. Unlike other low exchange energy systems, substantial oscillator strength is sustained at the singlet-triplet degeneracy point.
\end{abstract}

Main Text: Since the demonstration of thin-film OLEDs in the 1980s $(1,2)$, these devices have evolved from lab curiosity to global industry. The efficiency of an OLED is fundamentally governed by the spin of bound electron-hole pairs (excitons) which form through the recombination of injected electrons and holes. The ratio of emissive singlets to dark triplets formed from randomly spin-polarized electron and hole currents is 1:3, setting a limit of $25 \%$ internal quantum efficiency (IQE) for fluorescent OLEDs. The exchange energy (singlet-triplet energy gap) is generally large and positive, around $0.5 \mathrm{eV}$. Therefore, triplets cannot usually undergo intersystem crossing (ISC) to emissive singlets. Phosphorescent OLEDs, which utilize the heavy-atom effect in platinum or iridium compounds to render triplets emissive $(3,4)$, and thermally activated delayed fluorescence (TADF) OLEDs, based on thermally-promoted tripletto-singlet up-conversion for systems with low exchange energies (5), enable higher efficiencies. 
Here we show sub-microsecond luminescent harvesting of triplets in a new family of linear copper and gold carbene-metal-amides (CMAs). Importantly, this requires rapid ISC from the triplet state, which is enabled in this family of compounds at molecular configurations where the exchange energy is near zero, resulting in extremely efficient electroluminescence (EL).

We have recently shown that two-coordinate complexes of copper and gold based on cyclic (alkyl)(amino)-carbene ligands (CAACs) (6) can exhibit extremely high solid-state photoluminescence quantum efficiencies due to the lack of strong intermolecular interactions (7). To explore their use in OLEDs, we designed and synthesized CMA compounds (CAAC)AuCz (CMA1), (CAAC)CuCz (CMA2), (CAAC)AuNPh2 (CMA3), and (CAAC)AuDTBCz (CMA4) (Fig. 1A) $(\mathrm{Cz}=$ carbazole anion, $\mathrm{DTBCz}=3,6$-di-tert-butylcarbazole anion). They are soluble in a range of organic solvents, do not undergo ligand rearrangement, and are thermally-stable to $>270{ }^{\circ} \mathrm{C}$ (Table S1) (8). Their stability and solubility render them well-suited for solution processing, while their high decomposition temperatures allow the possibility of vacuum sublimation. Measured redox potentials are presented in Table S2 (8). Absorption spectra show a molecular charge-transfer band, with an increase in extinction coefficient from $\sim 5 \times 10^{3} \mathrm{M}^{-1} \mathrm{~cm}^{-1}$ at $400 \mathrm{~nm}$ to $>2 \times 10^{4} \mathrm{M}^{-1} \mathrm{~cm}^{-1}$ for the higher-lying localized absorption bands (Figs. 1B \& S1) (8).

To use this new class of emitter in OLEDs, we developed a multi-layer device structure with all organic layers solution-processed. A 180 nm polymeric hole-transport/electronblocking layer, poly(9,9-dioctylfluorene-co-N-(4-butylphenyl)diphenylamine) (TFB), was deposited on PEDOT:PSS from toluene solution without additional crosslinking (9), a $20 \mathrm{~nm}$ emissive layer comprising CMA1-4 dispersed as a guest emitter in a poly(9-vinylcarbazone) (PVK) host $(4,10)$ was deposited from dimethylformamide solution, while a $\sim 70 \mathrm{~nm}$ layer of 
bathophenanthroline (BPhen) electron-transport/hole-blocking layer was deposited from methanol solution. The device energetic structure is shown in Fig. 1C.

Fig. 1D presents the maximum-EQE histogram of 182 OLEDs using CMA4. EL spectra and external quantum efficiencies (EQEs) of the best OLEDs are shown in Fig. 1E and Table 1 (additional data in Figs. S2A \& S2B) (8). Angle-resolved EL profiles showed Lambertian emission (Fig. S2C), as is typical for OLEDs without microcavity outcoupling (11), allowing accurate estimation of EQE from on-axis irradiance. Commission International de l'éclairage (CIE) color coordinates showed no variation with EQE (Figs. S2D \& S2E). The EQEs of the best devices at practical luminance $\left(100 \& 1000 \mathrm{~cd} \mathrm{~m}^{-2}\right)$ are $>25 \%$. While the precise structural control offered by vacuum-sublimation usually enables superior performance, the metrics of our solution-processed devices are comparable to, or exceed, those of state-of-the-art OLEDs $(5,12)$ and quantum-dot LEDs (13). At brightness suitable for display applications, we achieve the highest quantum efficiencies reported for any solution-processed LEDs without enhanced optical outcoupling $(13,14)$. Assuming outcoupling efficiencies of 20-30\% for planar OLEDs $(5,11$, 13), we infer a close-to-unity (80-100\%) IQE for the best devices (using CMA1\&4).

Time-resolved EL measurements show that emission occurs entirely via a sub- $\mu \mathrm{s}$ delayed-emission channel (Figs. 1B\&1F) and there is no prompt (ns) component which would usually distinguish between geminate singlets and triplets $(15,16)$. These imply that all excitons, regardless of initial spin, contribute to luminescence. The EL lifetime decreases with increasing current density, likely due to bimolecular processes (17).

To understand the emission mechanism of CMAs, we measure transient photoluminescence (PL) and absorption (TA) spectra of spin-cast films. All compounds show a $<1 \mathrm{~ns}$ prompt-PL component, and a delayed component whose lifetime is strongly temperature- 
dependent (Fig. 2A). Time-resolved PL spectra of CMA1 on ns- $\mu$ s timescales at 300K (Fig. 2B) show a red-shift of the PL peak between prompt and delayed emission from $\sim 490 \mathrm{~nm}$ at $0-2 \mathrm{~ns}$, to $\sim 540 \mathrm{~nm}$ at later times. The prompt emissive species is therefore spectrally distinct from the delayed emissive species. At $300 \mathrm{~K}$, the delayed emission accounts for $>99 \%$ of the PL, and is reversibly quenched by oxygen (Fig. S3) (8).

Fig. 2C presents ultrafast (fs-ps) PL (18) of CMA1 neat films at 300K. Emission is characterized by a single component with 4 ps lifetime. Its initial peak at $470 \mathrm{~nm}$ shifts to 480 $\mathrm{nm}$ within 500 fs and then stabilizes, consistent with the 0-2 ns prompt component identified in Fig. 2B. ps- $\mu$ s TA measurements (Fig. 2D) show the initial photo-induced absorption (PIA) near $680 \mathrm{~nm}$, from the singlet exciton, converts to a single long-lived species on the same time-scale as the initial PL decay (Fig. 2G), which we assign to triplets. The triplet possesses two other covarying PIA features in the near-infrared (Fig. S4) (8). Both singlet and triplet PIA spectra agree semi-quantitatively with calculated excited-state transitions (Fig. S5 and Table S6) (8). The triplet PIA lifetime is $\sim 300 \mathrm{~ns}$ at $300 \mathrm{~K}$ (Fig. 2D). We infer that ISC to triplets occurs within 4 ps after photoexcitation, quenching the prompt fluorescence, such that the majority of excitations pass through the triplet manifold. Triplets thus act as the reservoir for delayed emission.

The delayed emission exhibits two distinct regimes. Below 100K, it peaks at $\sim 510 \mathrm{~nm}$ with $\sim 10 \mu$ s lifetime, independent of temperature (Figs. 2A \& 2B), which we assign to phosphorescence. This phosphorescence redshifts by $\sim 5 \mathrm{~nm}$ over the first $3 \mu \mathrm{s}$, consistent with diffusion of triplets to lower-energy sites. Above 100K, the lifetime of the delayed component decreases, dropping to $\sim 350 \mathrm{~ns}$ at $300 \mathrm{~K}$ (Fig. 2A), while the total PL intensity increases (Fig. S6A) (8). The $300 \mathrm{~K}$ delayed emission is $\sim 150 \mathrm{meV}$ lower in energy than the $4 \mathrm{~K}$ phosphorescence. The characteristic activation energy $\left(E_{\mathrm{A}}\right)$ of the temperature-dependent 
emission is $45 \mathrm{meV}$ for CMA1 (Fig. S6B). $E_{\mathrm{A}}$ and emission lifetimes for CMA2-4 are similar (Fig. S7) (8). A physical model for $E_{\mathrm{A}}$ is outlined in Supplementary Materials (8). The PL spectra of the delayed emission in host-guest films are nearly identical to the steady-state EL spectra in devices (Fig. 1B). EL spectra typically correspond to the lowest-accessible excited state, where recombination preferentially occurs. We consider that at $300 \mathrm{~K}$, triplets formed electrically or after ISC from photogenerated singlets contribute to emission at a rate of $\sim 3 \times 10^{6}$ $\mathrm{s}^{-1}$. This remarkably rapid utilization of triplets via the delayed-emission channel enables CMAbased OLEDs to maintain efficient operation at high brightness. Identifying its origin is therefore crucial to understanding their high performance.

To establish the nature of the low-energy delayed emission, we turn to the model system of molecular emitters in dilute solution, in which intermolecular interactions are suppressed and structural reorganization is unhindered. Ultrafast PL spectra for CMA1 in chlorobenzene solution (Fig. 2E) show that the PL peak shifts from $470 \mathrm{~nm}$ to $510 \mathrm{~nm}$ within $5 \mathrm{ps}$ (Fig 2F). This spectral evolution is independent of solvent polarity (Fig. S8) (8). The bulk of the steady-state emission in solution occurs via the delayed pathway, peaked at $\sim 550 \mathrm{~nm}$ (Fig. 1B). In solution, we therefore observe ps geometrical relaxation of the photoexcited molecule which is coupled to the singlet energy.

Raman spectroscopy of CMA1 identifies a cluster of modes around $20-30 \mathrm{~cm}^{-1}$ with periods close to the observed relaxation time (Fig. S9) (8). Density functional theory (DFT) calculations identify these as relative rotation of donor and acceptor about the metal linkage. Their Raman activity is $70 \%$ of the strength of the cluster of modes around $1500 \mathrm{~cm}^{-1}$, originating from $\mathrm{C}-\mathrm{N}$ and $\mathrm{C}=\mathrm{C}$ ring-stretches on the donor and acceptor moieties. Raman modes mediating electronic transitions that change the polarizability of the molecule, such as the $S_{0} \rightarrow$ 
$S_{l}$ transition, show enhanced activity (50). These include ring-stretching modes in $\pi$-delocalized systems, which couple to a Peierls distortion. The strong Raman activity we observe for lowfrequency rotational modes is unusual, and implies that low-energy rotations mediate transitions between an excited singlet-state and the ground-state. By contrast, the analogous rotation of the substituted phenyl of CMA1 (48-52 $\left.\mathrm{cm}^{-1}\right)$ has considerably suppressed Raman activity, owing to its weak coupling to the $S_{0} \rightarrow S_{1}$ transition. The large shift in singlet energy observed over the first few ps in luminescence spectra in solution is therefore consistent with a rotational relaxation of the molecule about the metal-amide bond. Similar behavior accounts for the dual-fluorescence observed in twisted-intramolecular charge-transfer (TICT) emitters $(19,20)$, for which relaxation to a twisted geometry is sufficiently slow that luminescence from intermediate geometries can be observed. In solid films (Figs. 2C\&2F) this early-time redshift is absent, consistent with largescale reorganization, such as rotation, being suppressed in the solid state.

We consider that at temperatures above $100 \mathrm{~K}$, the sub- $\mu$ s delayed emission in solid films is delayed fluorescence, rather than phosphorescence (which has $\sim 10 \mu$ s lifetime below 100K), with reverse ISC and radiative recombination of singlets as the rate-limiting steps. Prompt fluorescence is outcompeted by ISC to triplets, which occurs in $\sim 4 \mathrm{ps}$. This is consistent with the majority of both photo- and electrical-excitations passing through the triplet manifold before emission, as discussed. We note that, during geometric relaxation in solution, singlet emission with energies above, below and equal to that of the measured phosphorescence is observed, indicating that geometries exist at which singlet and triplet energies approach degeneracy.

To understand the link between molecular geometry and the energetic ordering of singlet and triplet states, the energies and optimized molecular structures of the ground $\left(S_{0}\right)$, first excited singlet $\left(S_{1}\right)$ and first excited triplet $\left(T_{1}\right)$ states for CMA1-4 were calculated using DFT and time- 
dependent DFT (TD-DFT). Ground-state calculations yield close agreement with X-ray crystal structures (Fig. S10 and Table S3) (8). For all compounds, $S_{0}$ and the relaxed $T_{1}$ states correspond to a geometry with co-planar carbene and amide ligands (denoted by superscript $P$ ), while in the relaxed $S_{1}$ state the amide ligand is rotated by $90^{\circ}$ relative to the carbene (denoted by superscript $R$ ) (Figs. 3A \& S11A) (8). Both $S_{1}$ and $T_{1}$ have strong CT character, with excitation from the highest-occupied molecular orbital (HOMO), mostly localized on the amide, to the lowest-unoccupied molecular orbital (LUMO), mostly localized on the carbene, contributing over $90 \%$ of the $S_{0} \rightarrow S_{1}$ transition (Figs. 3B \& S11B). Extended conjugation through the metal in the co-planar geometry is consistent with CT states contributing significantly to absorption (Figs. 1B and S1). $T_{1}^{P}$ lies lower in energy than its co-planar singlet counterpart, $S_{1}^{P}$. However, increasing the dihedral angle allows $S_{1}$ to stabilize relative to $T_{1}$. Upon full relaxation, $S_{1}^{R}$ is lower in energy than $T_{1}^{P}$, leading to a negative $S_{1}^{R}-T_{1}^{P}$ energy gap $\left(\Delta E_{\mathrm{ST}}<0\right)$, which we term "rotationally accessed spin-state inversion" (RASI) (Fig. 3C). During relaxation from $S_{1}^{P}$ to $S_{1}^{R}$, the singlet energy therefore passes through the triplet energy, in agreement with PL data. For CMA1, the calculated energy barrier for a full rotation of the metal-amide bond in the ground state is $143 \mathrm{meV}$, equivalent to the energy of a freely-rotatable $\mathrm{C}-\mathrm{C}$ bond (21). We therefore consider relative rotation of the carbene and amide groups about the metal-amide bond to be the singlet relaxation mode identified by spectroscopy.

The stabilization of $S_{l}$ by rotation leads to a change of sign of the exchange energy at an intermediate dihedral angle $\theta_{0}$. We consider that the rapid reverse ISC required to explain both the high delayed fluorescence rate and the high device IQE is achieved at molecular geometries close to $\theta_{0}$ (Fig. 3C). ISC is likely enhanced by metal-assisted spin-orbit coupling. For CMA1, 
we estimate $\theta_{0}$ to be $\sim 45^{\circ}$ (Fig. S12) (8), after empirically correcting for calculation errors. The calculated oscillator strength for $\mathrm{S}_{0} \rightarrow \mathrm{S}_{1}$ falls to approximately zero at $\theta=90^{\circ}$ but at the singlettriplet degeneracy point, is around half of the $0^{\circ}$ value, yielding a transition with characteristic extinction coefficient $>10^{3} \mathrm{M}^{-1} \mathrm{~cm}^{-1}$ (Fig. S12).

TD-DFT calculations suggest that in the CMA material family, RASI arises from a stronger electrostatic attraction between electron and hole in the rotated singlet geometry, enabled by the localization of electron density on the M-C bond. In the triplet state, the more delocalized electron density at this bond leads to a weaker attraction, insufficient to overcome the torsional potential associated with increasing the dihedral angle. Electron density maps are presented in Fig. S13 (8). This behavior contrasts with carbene-metal-halide (CMH) molecules involving the same carbene ligands (7). For CMHs, the calculated $\Delta E_{\mathrm{ST}}$ was $300-400 \mathrm{meV}$, too large to allow reverse ISC. The behavior of the CMAs also contrasts with 3-coordinate $\mathrm{Cu}(\mathrm{I})$ compounds where rotational freedom does not allow spin-state inversion (22).

Combined, these data provide a framework for understanding the luminescence behavior of CMA emitters in different material phases. Fig. 3D presents a schematic of the steady-state PL peak energies of CMA1 in phases with differing access to rotated states. The highest PL energy $(2.6 \mathrm{eV})$ is observed for polycrystalline powders, where all molecules are constrained into a nearcoplanar configuration (Fig. S10). Spin-cast dilute (1.5 wt\%) host-guest films show $2.45 \mathrm{eV}$ emission; more concentrated (20\%) host-guest films emit at $2.4 \mathrm{eV}$, while neat films emit at $\sim 2.3$ $\mathrm{eV}$. In the spin-cast amorphous phase, molecules deposited at room-temperature will deviate stochastically from the ground-state geometry and exist as a mixture of rotamers, consistent with photothermal deflection spectroscopy of CMA1 neat films showing a broad Urbach tail (Fig. S1). Diffusion to a range of rotated states is thus easier in spin-cast films than in crystal, and 
depends on the local density of emitters. Dynamic access to different molecular configurations may also be possible. In the solution phase, molecules dynamically reorganize to the lowestenergy configuration; steady-state emission in solution is at $\sim 2.2 \mathrm{eV}$. We therefore observe a direct correlation between the availability of rotated molecular geometries and the energy of emission, whether such geometries are accessed dynamically or via diffusion.

We have synthesized a new class of materials for high-performance OLEDs exhibiting rapid emission via the triplet state, such that all electron-hole recombination events can lead to luminescence. At $300 \mathrm{~K}$, the emission lifetimes for CMA1 is $350 \mathrm{~ns}$, considerably faster than that achieved to date in efficient iridium-based phosphorescent emitters (23-25) (typical lifetimes $>1.5 \mu \mathrm{s}$ ) and efficient TADF emitters (typical lifetimes $>5 \mu \mathrm{s})(5,26)$. This is possible due to the existence of molecular configurations which achieve low exchange energies while still retaining appreciable oscillator strength, in contrast to TADF materials for which low exchange energy is achieved at the expense of oscillator strength (27). Sub-microsecond emission is particularly beneficial for efficient high-brightness OLED operation, as we demonstrate, and for avoiding degradation pathways arising from bimolecular annihilation events (17).

\section{References and Notes:}

1. C. Tang, S. VanSlyke, Organic electroluminescent diodes. Appl. Phys. Lett. 51, 913 (1987).

2. J. Burroughes et al., Light-emitting diodes based on conjugated polymers, Nature 347, 539541 (1990).

3. M. Baldo et al., Highly efficient phosphorescent emission from organic electroluminescent devices, Nature 395, 151-154 (1998).

4. Y. Ma, H. Zhang, J. Shen, C. Che, Electroluminescence from triplet metal-ligand charge- 
transfer excited state of transition metal complexes, Synthetic Metals 94, 245-248 (1998).

5. H. Uoyama, K. Goushi, K. Shizu, H. Nomura, C. Adachi, Highly efficient organic lightemitting diodes from delayed fluorescence, Nature 492, 234-238 (2012).

6. G. Frey, R. Dewhurst, S. Kousar, B. Donnadieu, G. Bertrand, Cyclic (alkyl)(amino)carbene gold(I) complexes: A synthetic and structural investigation, J. Organomet. Chem. 693, 16741682 (2008).

7. A. Romanov et al., Highly photoluminescent copper carbene complexes based on prompt rather than delayed fluorescence, Chem. Commun. 52, 6379-6382 (2016).

8. Full details can be found in the supplementary materials on Science Online.

9. R. Png et al., High-performance polymer semiconducting heterostructure devices by nitrenemediated photocrosslinking of alkyl side chains, Nat. Mater. 9, 152-158 (2009).

10. D. Abbaszadeh et al., Elimination of charge carrier trapping in diluted semiconductors, Nat. Mater. 15, 628-633 (2016).

11. N. Greenham, R. Friend, D. Bradley, Angular Dependence of the Emission from a Conjugated Polymer Light-Emitting Diode: Implications for efficiency calculations, Adv. Mater. 6, 491494 (1994).

12. S. Reineke et al., White organic light-emitting diodes with fluorescent tube efficiency, Nature 459, 234-238 (2009).

13. X. Dai et al., Solution-processed, high-performance light-emitting diodes based on quantum dots, Nature 515, 96-99 (2014).

14. N. Aizawa et al., Solution-processed multilayer small-molecule light-emitting devices with high-efficiency white-light emission, Nat. Commun. 5, 5756 (2014).

15. D. Kondakov, Characterization of triplet-triplet annihilation in organic light-emitting diodes 
based on anthracene derivatives, J. Appl. Phys. 102, 114504 (2007).

16. B. Wallikewitz, D. Kabra, S. Gélinas, R. Friend, Triplet dynamics in fluorescent polymer lightemitting diodes, Phys. Rev. B 85, 045209 (2012).

17. N. Giebink, S. Forrest, Quantum efficiency roll-off at high brightness in fluorescent and phosphorescent organic light emitting diodes, Phys. Rev. B 77, 235215 (2008).

18. K. Chen, J. Gallaher, A. Barker, J. Hodgkiss, Transient Grating Photoluminescence Spectroscopy: An Ultrafast Method of Gating Broadband Spectra, J. Phys. Chem. Lett. 5, $1732-1737$ (2014).

19. S. Sasaki, G. Drummen, G. Konishi, Recent advances in twisted intramolecular charge transfer (TICT) fluorescence and related phenomena in materials chemistry, J. Mater. Chem. C 4, 2731-2743 (2016).

20. A. Siemiarczuk, Z. Grabowski, A. Krówczyński, M. Asher, M. Ottolenghi, Two emitting states of excited p-(9-anthryl)-n,n-dimethylaniline derivatives in polar solvents, Chem. Phys. Lett. 51, 315-320 (1977).

21. J. Zheng, K. Kwak, J. Xie, M. Fayer, Ultrafast Carbon-Carbon Single-Bond Rotational Isomerization in Room-Temperature Solution, Science 313, 1951-1955 (2006).

22. M. Leitl, V. Krylova, P. Djurovich, M. Thompson, H. Yersin, Phosphorescence versus thermally activated delayed fluorescence. Controlling singlet-triplet splitting in brightly emitting and sublimable $\mathrm{Cu}(\mathrm{I})$ compounds. J. Am. Chem. Soc. 45, 16032-16038 (2014).

23. K. Goushi, Y. Kawamura, H. Sasabe, C. Adachi, Unusual Phosphorescence Characteristics of Ir(ppy) ${ }_{3}$ in a Solid Matrix at Low Temperatures, Jpn. J. Appl. Phys. 43, L937-L939 (2004).

24. T. Sajoto et al., Temperature Dependence of Blue Phosphorescent Cyclometalated Ir(III) Complexes, J. Am. Chem. Soc. 131, 9813-9822 (2009). 
25. M. Baldo, S. Lamansky, P. Burrows, M. Thompson, S. Forrest, Very high-efficiency green organic light-emitting devices based on electrophosphorescence, Appl. Phys. Lett. 75, 4 (1999).

26. Y. Tao et al., Thermally Activated Delayed Fluorescence Materials Towards the Breakthrough of Organoelectronics, Adv. Mater. 26, 7931-7958 (2014).

27. R. Gómez-Bombarelli et al., Design of efficient molecular organic light-emitting diodes by a high-throughput virtual screening and experimental approach, Nat. Mater. 15, 1120-1127 (2016).

28. A. Romanov, M. Bochmann, Gold(I) and Gold(III) Complexes of Cyclic (Alkyl)(amino)carbenes, Organometallics 34, 2439-2454 (2015).

29. G. Gritzner, J. Kůta, Recommendations on reporting electrode potentials in nonaqueous solvents, Electrochim. Acta 29, 869-873 (1984).

30. Programs CrysAlisPro, Oxford Diffraction Ltd., Abingdon, UK (2010).

31. G. Sheldrick, A short history of SHELX, Acta Cryst Sect A 64, 112-122 (2007).

32. F. Furche, D. Rappoport, Density functional methods for excited states: equilibrium structure and electronic spectra in Computational Photochemistry (ed. M. Olivuccim) 93-128 (Elsevier, Amsterdam, 2005).

33. J. Perdew, K. Burke, M. Ernzerhof, Generalized Gradient Approximation Made Simple, Phys. Rev. Lett. 77, 3865-3868 (1996).

34. C. AdamoV. Barone, Toward reliable density functional methods without adjustable parameters: The PBE0 model, J. Chem. Phys. 110, 6158 (1999).

35. F. Weigend, M. Häser, H. Patzelt, R. Ahlrichs, RI-MP2: optimized auxiliary basis sets and demonstration of efficiency, Chem. Phys. Lett. 294, 143-152 (1998). 
36. F. Weigend, R. Ahlrichs, Balanced basis sets of split valence, triple zeta valence and quadruple zeta valence quality for $\mathrm{H}$ to Rn: Design and assessment of accuracy, Phys. Chem. Chem. Phys. 7, 3297 (2005).

37. K. Peterson, D. Figgen, E. Goll, H. Stoll, M. Dolg, Systematically convergent basis sets with relativistic pseudopotentials. II. Small-core pseudopotentials and correlation consistent basis sets for the post-d group 16-18 elements, J. Chem. Phys. 119, 11113 (2003).

38. D. Andrae, U. Haeussermann, M. Dolg, H. Stoll, H. Preuss, Energy-adjustedab initio pseudopotentials for the second and third row transition elements, Theor. Chim. Acta 77, 123$141(1990)$.

39. M. Bühl, C. Reimann, D. Pantazis, T. Bredow, F. Neese, Geometries of Third-Row Transition-Metal Complexes from Density-Functional Theory, J. Chem. Theory Comput. 4, $1449-1459$ (2008).

40. R. Kang, H. Chen, S. Shaik, J. Yao, Assessment of Theoretical Methods for Complexes of Gold(I) and Gold(III) with Unsaturated Aliphatic Hydrocarbon: Which Density Functional Should We Choose?, J. Chem. Theory Comput. 7, 4002-4011 (2011).

41. C. Adamo, G. Scuseria, V. Barone, Accurate excitation energies from time-dependent density functional theory: Assessing the PBE0 model, J. Chem. Phys. 111, 2889 (1999).

42. C. Adamo, V. Barone, R. Subra, The mechanism of spin polarization in aromatic free radicals, Theoretical Chemistry Accounts: Theory, Computation, and Modeling (Theor. Chim. Acta) 104, 207-209 (2000).

43. D. Jacquemin, A. Planchat, C. Adamo, B. Mennucci, TD-DFT Assessment of Functionals for Optical 0-0 Transitions in Solvated Dyes, J. Chem. Theory Comput. 8, 2359-2372 (2012). 
44. A. Laurent, M. Medved, D. Jacquemin, Using TD-DFT to probe the nature of donor-acceptor Stenhouse adduct (DASA) photochromes, ChemPhysChem, DOI: 10.1002/cphc.201600041 (2016).

45. I. Koshevoy et al., Intensely Luminescent Alkynyl-Phosphine Gold(I)-Copper(I) Complexes: Synthesis, Characterization, Photophysical, and Computational Studies, Inorg. Chem. 48, 2094-2102 (2009).

46. I. Koshevoy et al., Luminescent Gold(I) Alkynyl Clusters Stabilized by Flexible Diphosphine Ligands, Organometallics 33, 2363-2371 (2014).

47. Y. Zhao, D. Truhlar, The M06 suite of density functionals for main group thermochemistry, thermochemical kinetics, noncovalent interactions, excited states, and transition elements: two new functionals and systematic testing of four M06 functionals and 12 other functionals, Theor Chem Account 119, 525-525 (2008).

48. TURBOMOLE V6.3 2011, a development of University of Karlsruhe and Forschungszentrum Karlsruhe GmbH, 1989 -2007, TURBOMOLE GmbH, since 2007; available from http://www.turbomole.com.

49. M. Frisch et al., Gaussian 09, Revision C.01, Gaussian, Inc., Wallingford, CT (2010).

50. E. Ehrenfreund, Z. Vardeny, O. Brafman, B. Horovitz, Amplitude and phase modes intranspolyacetylene: Resonant Raman scattering and induced infrared activity, Phys. Rev. B 36, 1535-1553 (1987). 
Acknowledgments: We thank Dr. M. Roberts and Cambridge Display Technology Ltd.

(Company No. 02672530) for re-calibration of OLED measurements and for angular emission tests. We thank Dr. A. Morris, Dr. H. Stern, Dr. A. Sadhanala, B. Zhu, L. Meraldi, J.

Ratanapreechachai and J. Nisbett for help and discussions. D.D. and R.H.F. acknowledge the Department of Physics (Cambridge) and the KACST-Cambridge University Joint Centre of Excellence for support. L.Y. thanks the Singapore Agency for Science, Technology and Research (A*STAR) for PhD studentship. M.L. acknowledges support by the Academy of Finland (Project 251448). The computations were made possible by use of the Finnish Grid Infrastructure and Cloud Infrastructure. This work was supported by the European Research Council. M.B. is an ERC Advanced Investigator Award holder (Grant No. 338944-GOCAT). D.C. and S.J. acknowledge the Royal Society for support. CCDC-1472605 (CMA1), CCDC1472607 (CMA2), CCDC-1472606 (CMA3) and CCDC-1473622 (CMA4) contain supplementary crystallographic data, which can be obtained free of charge from the Cambridge Crystallographic Data Centre via www.ccdc.cam.ac.uk/data_request/cif. 
A

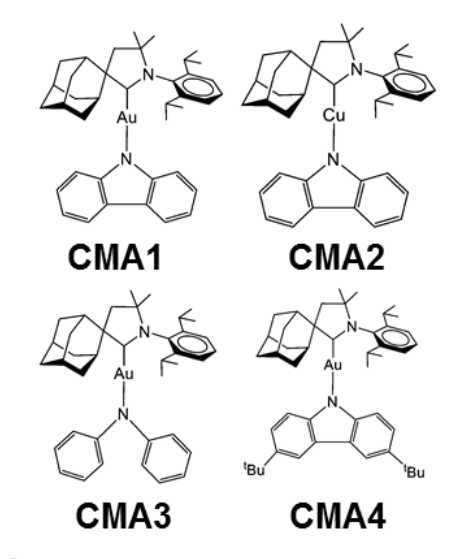

C

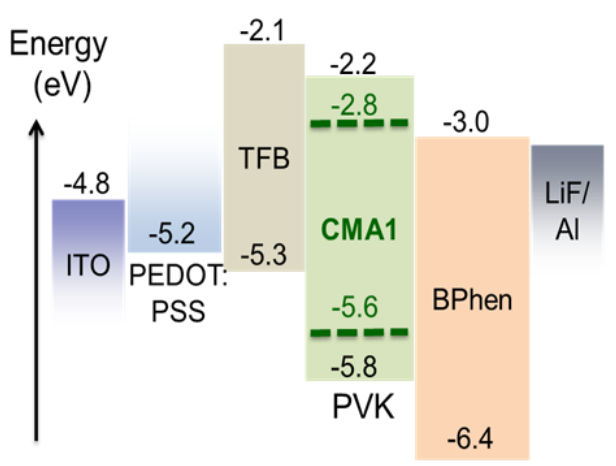

E

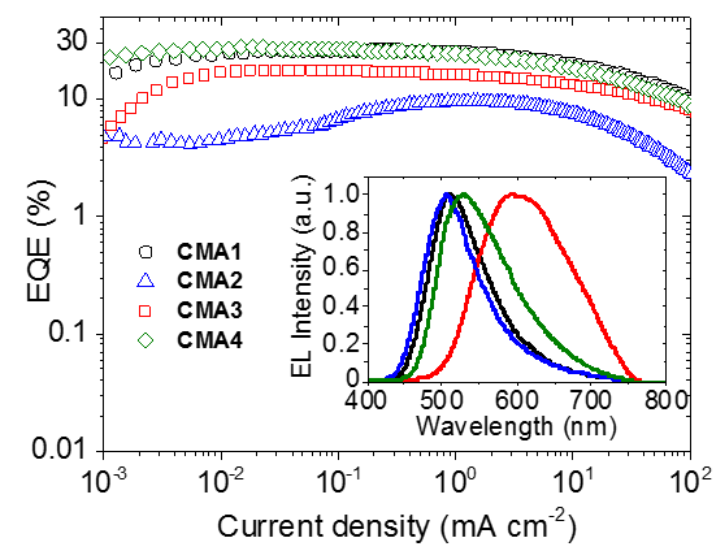

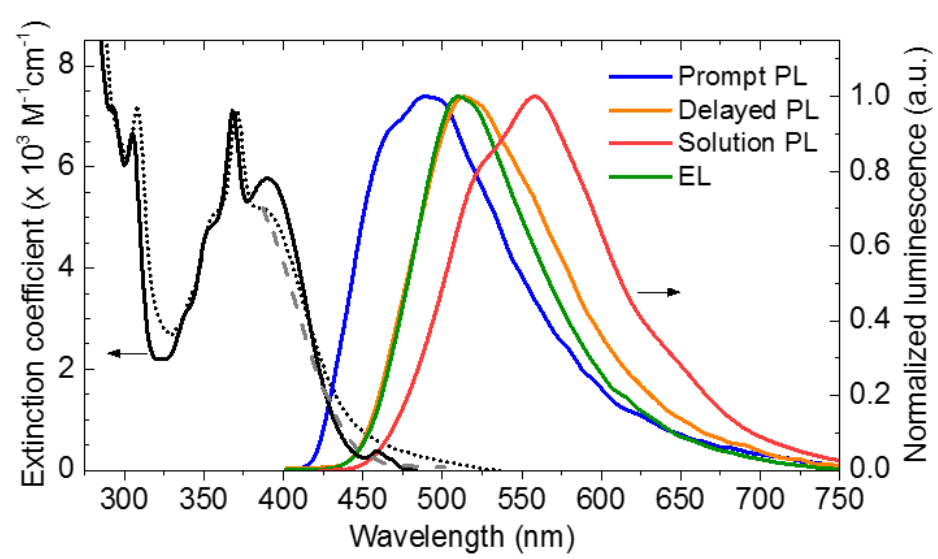

D
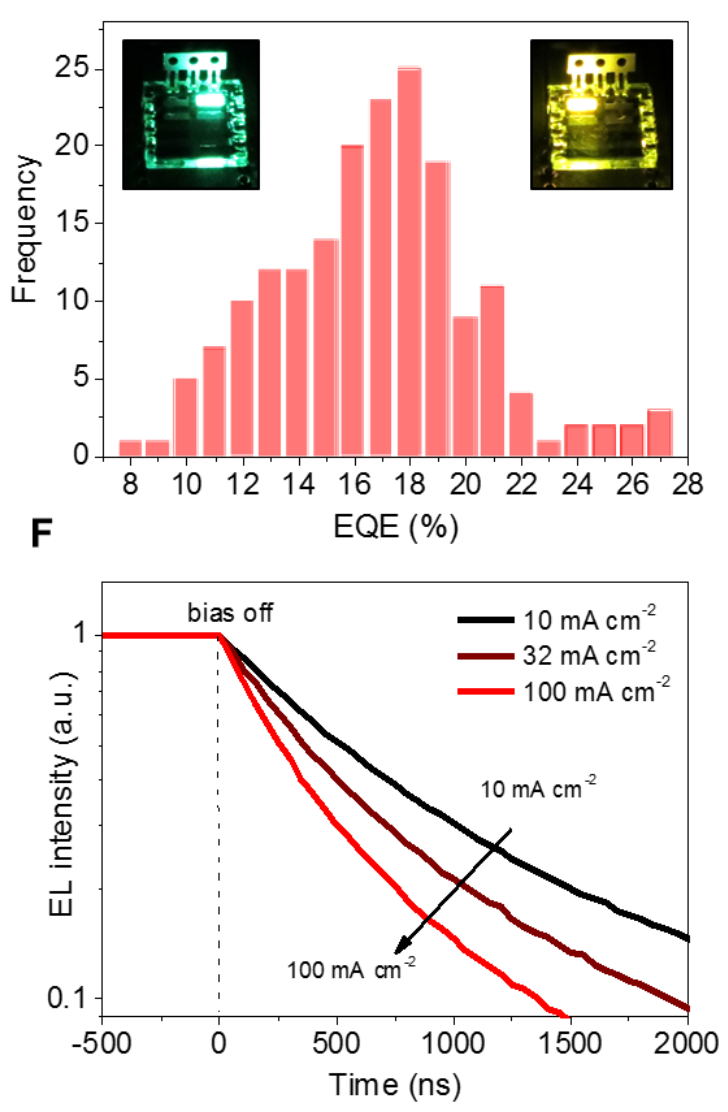

Fig. 1 Chemical structures, absorption/emission spectra and OLED performance. (A)

Chemical structures of CMAs. (B) (Left axis) Molar extinction coefficients of CMA1 in THF solution (solid) and absorption in neat film (dot). Overlaid photothermal deflection spectrum (dash) indicates the film absorption tail arises primarily from scattering. To convert from molar 
extinction $\left(\mathrm{M}^{-1} \mathrm{~cm}^{-1}\right)$ to film absorption ( $\left.\mathrm{cm}^{-1}\right)$, multiply by 2. (Right axis) PL and EL spectra of spin-cast films of CMA1 dispersed in PVK host (at $20 \mathrm{wt} \%$ ), and in solution (C) Energy level diagram of a CMA1 device. (D) Peak-EQE Histogram of 182 CMA4 devices (Most typical = $\sim 18 \%$; highest $=27.5 \%$ ). Insets: photographs of CMA1 (left) and CMA3 (right) devices. Device area $=5.25 \mathrm{~mm}^{2}$. (E) EQE curves and EL spectra (inset) for devices utilizing CMA1-4 dispersed at $20 \mathrm{wt} \%$ in PVK. (F) Transient-EL curves measured after holding a CMA1 device at various steady-state current densities. 
A

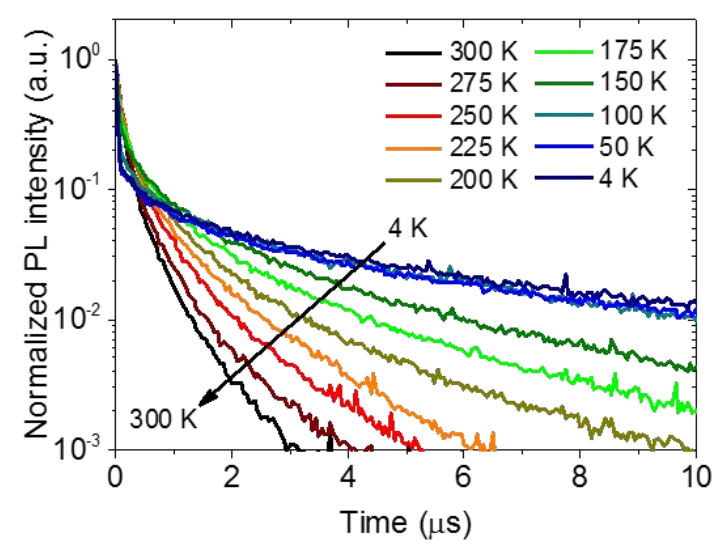

C

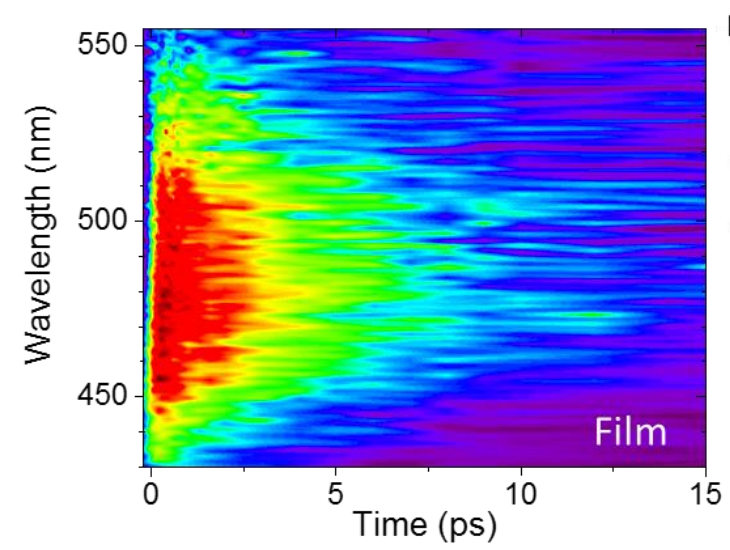

D

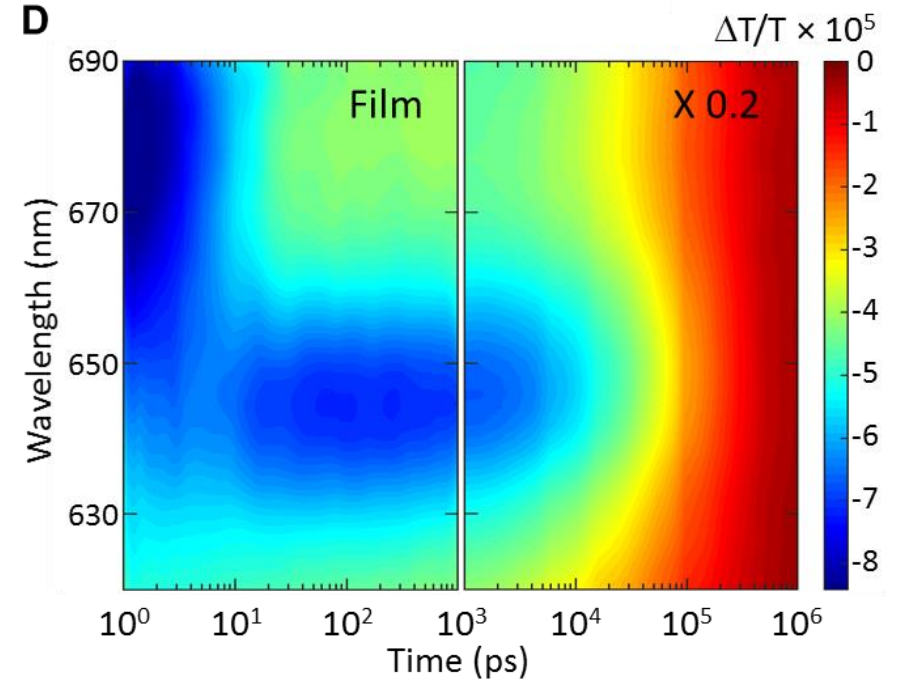

B

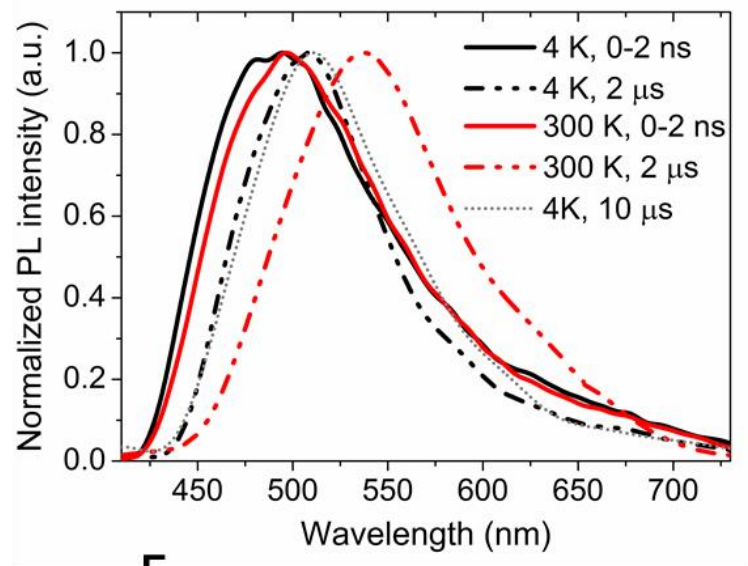

E

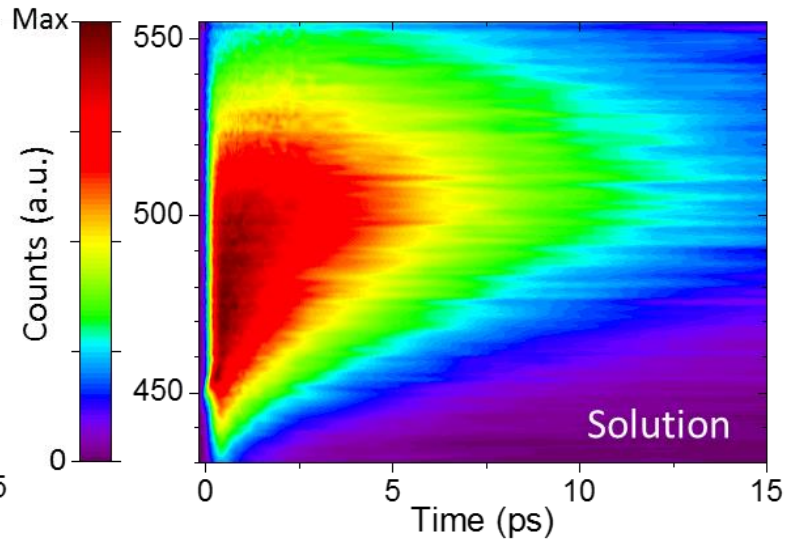

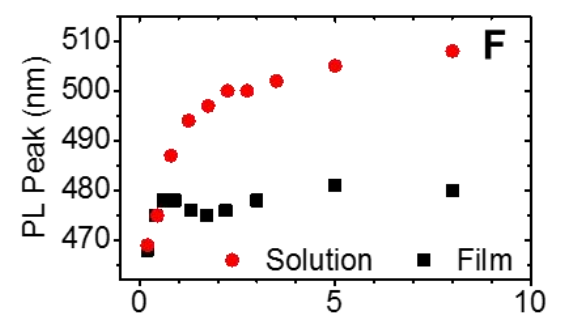

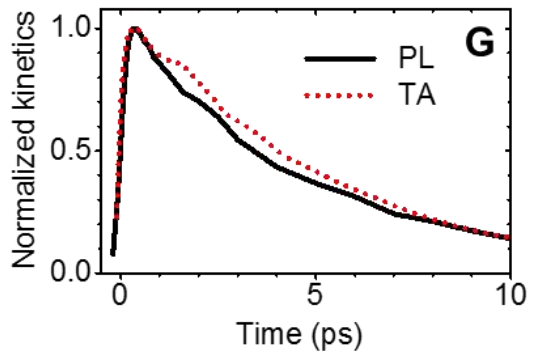

Fig. 2 Transient photophysical characterization of CMA1. (A) Temperature-dependent ns- $\mu \mathrm{s}$ PL kinetics measured using an electrically gated ICCD. (B) Prompt $(\mathrm{t}<2 \mathrm{~ns})$ and delayed $(\mathrm{t}=2$ $\mu \mathrm{s})$ PL spectra at $300 \mathrm{~K}$. The $10-\mu$ s spectrum at $4 \mathrm{~K}$ highlights the slight evolution of the 
phosphorescence spectrum. (C) Ultrafast Kerr-gated PL measurement of CMA1 neat film (300K), color scale indicates total counts above background. (D) TA spectra of CMA1 neat film (300K) on ps- $\mu$ s timescales. The initial excited state absorption associated with the singlet is at $680 \mathrm{~nm}$. The narrower blue-shifted band centered at $645 \mathrm{~nm}$ is associated with the triplet. (E) Ultrafast PL measurement of CMA1 (0.1 wt\%) in chlorobenzene (300K). (F) Evolution of PL peak position for the first $10 \mathrm{ps}$ of the ultrafast-PL decays. The solution sample exhibits a substantial red shift on this time-scale, which is absent in the solid film. (G) Decay kinetics of spectrally-integrated PL intensity and the 680 nm PIA in neat CMA1 films. Both share a common 4-ps decay time and the triplet PIA at $645 \mathrm{~nm}$ grows with the same timescale. All samples pumped at $400 \mathrm{~nm}$. 
A



$\mathrm{S}_{0}$

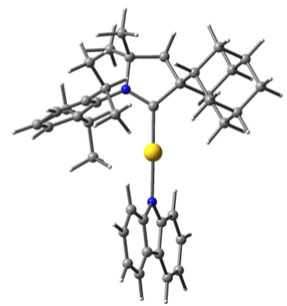

$\mathrm{S}_{1}$

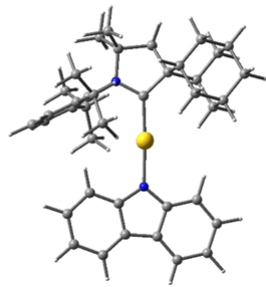

$\mathrm{T}_{1}$
B

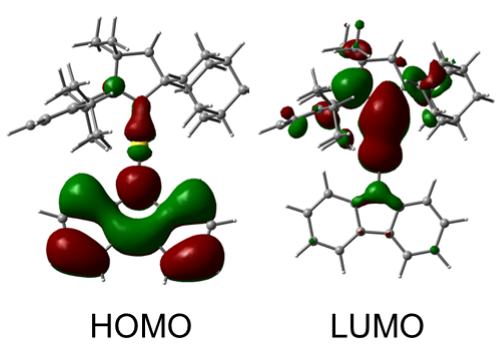

D

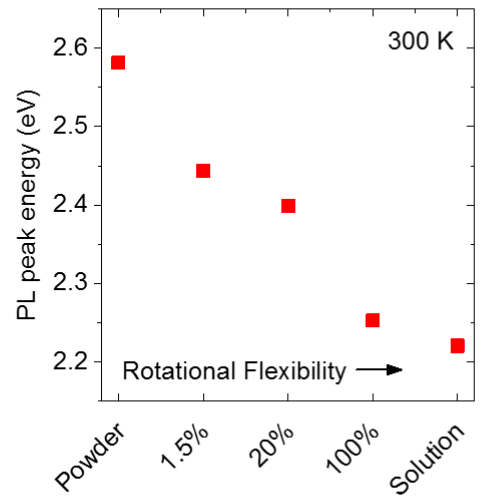

Dihedral angle (degrees)

Fig. 3 DFT calculations and the RASI mechanism. (A) Optimized geometries of CMA1 in $S_{0}$, $S_{1}$ and $T_{1}$ states. (B) HOMO and LUMO wavefunctions of CMA1 from DFT and TD-DFT calculations, isovalue $=0.02\left(\text { electrons } / \mathrm{bohr}^{3}\right)^{1 / 2}$. (C) Schematic of the RASI mechanism based on TD-DFT calculations, illustrating the stabilization of $\mathrm{S}_{1}$ and destabilization of $\mathrm{T}_{1}$ energies as the dihedral angle between donor and acceptor increases from co-planar $(\mathrm{P})$ to rotated $(\mathrm{R})$ geometries. Oscillator strength for the $\mathrm{S}_{1}$ to the ground state varies approximately sinusoidally with dihedral angle. $\mathrm{S}_{1}-\mathrm{T}_{1}$ degeneracy occurs at angle $\theta_{0}$. Trajectories for photogenerated singlets (dot) and electrically-generated triplets (dash) are indicated. (D) Steady-state PL peak energies of CMA1 in (from left to right) polycrystalline powder, $1.5 \mathrm{wt} \%$ in PVK, $20 \mathrm{wt} \%$ in PVK, neat film $(100 \mathrm{wt} \%)$ and $0.1 \mathrm{wt} \%$ in chlorobenzene. 
Table 1: Summary of OLED performances (best devices). Efficiencies at different brightness $\left(100 \mathrm{~cd} \mathrm{~m}^{-2}\right.$ and $\left.1000 \mathrm{~cd} \mathrm{~m}^{-2}\right)$ are shown.

\begin{tabular}{|c|c|c|c|c|c|}
\hline \multirow{2}{*}{ Emitter } & \multirow{2}{*}{$\begin{array}{c}\text { Turn-on } \\
\text { Voltage } \\
\text { (V) }\end{array}$} & EQE (\%) & $\begin{array}{c}\text { Current Efficiency } \\
\quad\left(\text { cd A } A^{-1}\right)\end{array}$ & $\begin{array}{l}\text { Power Efficiency } \\
\quad\left(\operatorname{lm} \mathbf{W}^{-1}\right)\end{array}$ & \multirow{2}{*}{$\begin{array}{c}\text { Max. } \\
\text { Luminance } \\
\left(\mathbf{c d ~ m ^ { - 2 } )}\right.\end{array}$} \\
\hline & & $\begin{array}{l}\mathrm{Max} / \mathbf{1 0 0} / \mathbf{1 0 0 0} \\
\mathrm{cd} \mathrm{m}^{-2}\end{array}$ & $\begin{array}{c}\mathrm{Max} / \mathbf{1 0 0} / \mathbf{1 0 0 0} \\
\mathrm{cd} \mathrm{m}^{-2}\end{array}$ & $\begin{array}{c}\mathrm{Max} / \mathbf{1 0 0} / \mathbf{1 0 0 0} \\
\mathrm{cd} \mathrm{m}^{-2}\end{array}$ & \\
\hline CMA1 & 2.6 & $26.3 / 26.1 / 25.2$ & $76.3 / 75.8 / 73.0$ & $62.7 / 50.0 / 37.0$ & 44700 \\
\hline CMA2 & 3.4 & $9.7 / 8.9 / 9.2$ & $30.4 / 28.0 / 29.0$ & $11.8 / 11.7 / 9.3$ & 7790 \\
\hline CMA3 & 3.0 & $17.9 / 17.3 / 15.5$ & $45.2 / 43.7 / 39.1$ & $33.6 / 25.0 / 17.0$ & 39540 \\
\hline CMA4 & 2.6 & $27.5 / 26.6 / 24.5$ & $87.1 / 84.5 / 77.9$ & $75.1 / 50.2 / 35.5$ & 73100 \\
\hline
\end{tabular}


Supplementary Materials:

Materials and Methods

Supplementary Text

Figures S1-S14

Tables S1-S6

Author Contributions

References (28-50) 